J. Nonlinear Var. Anal. 5 (2021), No. 3, pp. 391-401

Available online at http://jnva.biemdas.com

https://doi.org/10.23952/jnva.5.2021.3.04

\title{
CONTINUOUS-TIME ERGODIC ALGORITHM FOR SOLVING MONOTONE VARIATIONAL INEQUALITIES
}

\author{
TRINH NGOC HAI \\ School of Applied Mathematics and Informatics, \\ Hanoi University of Science and Technology, 1 Dai Co Viet, Hai Ba Trung, Hanoi, Vietnam
}

\begin{abstract}
In this paper, we propose a new algorithm for solving monotone but not Lipschitz-continuous variational inequalities. We consider a projected-type dynamical system and prove that the solution of this dynamical system converges to a desired solution in an ergodic sense.
\end{abstract}

Keywords. Dynamical system; Variational inequality; Equilibrium problem; Ergodic algorithm.

\section{INTRODUCTION}

Let $C$ be a nonempty, closed and convex set in $\mathbb{R}^{m}$, and let $A: C \rightarrow \mathbb{R}^{m}$ be a mapping on $C$. The variational inequality of $A$ on $C$ is to

$$
\text { find } x^{*} \in C \text { such that }\left\langle A\left(x^{*}\right), y-x^{*}\right\rangle \geq 0 \quad \forall y \in C \text {. }
$$

In the past few years, this problem has become a hot topic in optimization. It is closely related to a series of other problems such as the fixed point problem, the optimal problem, the saddle point problem, the Nash equilibrium problem and so on. As a consequence, a lot of methods have been proposed for solving this problem [1]-[18]. Among them, the projected-type algorithm has the simplest form:

$$
\left\{\begin{array}{l}
x^{0} \in C, \\
x^{k+1}=P_{C}\left(x^{k}-\lambda_{k} A\left(x^{k}\right)\right) .
\end{array}\right.
$$

Although this algorithm is very simple, it converges under restrictive assumptions that $A$ is strongly pseudomonotone and Lipschitz continuous. In the case that $A$ is only monotone but is not Lipschitz continuous, the sequence $\left\{x^{k}\right\}$ generated by (1.1) is not convergent. In this case, the authors in [9] prove that the ergodic sequence of $\left\{x^{k}\right\}$, defined by

$$
z^{k}=\frac{\sum_{i=0}^{k} \lambda_{i} x^{i}}{\sum_{i=0}^{k} \lambda_{i}}
$$

converges to a solution of $\operatorname{VI}(A, C)$ provided that $\sum_{k=0}^{\infty} \lambda_{k}=\infty, \sum_{k=0}^{\infty} \lambda_{k}^{2}<\infty$ and

$$
\sum_{k=0}^{\infty} \lambda_{k}^{2}\left\|A\left(x^{k}\right)\right\|^{2}<\infty .
$$

E-mail address: hai.trinhngoc@ hust.edu.vn.

Received February 20, 2021; Accepted April 11, 2021.

(C)2021 Journal of Nonlinear and Variational Analysis 
The ergodic algorithm is one of the simplest methods for solving monotone, non-Lipschitz variational inequalities. It has been developed and extended in many papers [6, 19, 20].

Recently, dynamical systems have become an important tool for solving variational inequalities [21, 22, 23, 24, 25]. It is interesting to investigate the ergodic algorithm from a continuous time perspective. In this paper, we consider a dynamical system associated to (1.1). Under the assumption that $A$ is monotone, we prove that the solution of this dynamical system converges to the desired solution of $\operatorname{VI}(A, C)$ in an ergodic sense. Our new approach not only provides a new perspective on the ergodic algorithm but also removes condition (1.2). Also, we extend our results to equilibrium problems.

The rest of our paper is organized as follows. In the next section, we give some useful preliminaries. In Section 3, we introduce our method and its convergence analysis. Section 4 presents an extension of the new method to equilibrium problems.

\section{PRELIMinaries}

In this section, we provide some definitions, results and notations, which are needed in our later convergence analysis. We refer the reader to [11, 26, 27, 28] for more details. In the whole paper, we assume that $C$ is a nonempty, closed and convex set in $\mathbb{R}^{m}$. Let $x \in \mathbb{R}^{m}$. The projection of $x$ onto $C$ is defined by

$$
P_{C}(x):=\operatorname{argmin}\{\|z-x\|: z \in C\} .
$$

It is easy seen that the mapping $P_{C}($.$) is single-valued and well-defined.$

Proposition 2.1. [26] It holds that

(i) $\left\|P_{C}(x)-P_{C}(y)\right\| \leq\|x-y\| \forall x, y \in \mathbb{R}^{m}$,

(ii) $\left\langle y-P_{C}(x), x-P_{C}(x)\right\rangle \leq 0 \forall x \in \mathbb{R}^{m}, \forall y \in C$.

Definition 2.1. $[6,9]$

(i) A mapping $A: C \rightarrow \mathbb{R}^{m}$ is said to be monotone on $C$ iff

$$
\langle A(y)-A(x), y-x\rangle \geq 0 \forall x, y \in C .
$$

(ii) A bifunction $f: C \times C \rightarrow \mathbb{R}$ is said to be monotone on $C$ iff

$$
f(x, y)+f(y, x) \leq 0 \forall x, y \in C .
$$

Note that if the mapping $A: C \rightarrow \mathbb{R}^{m}$ is monotone on $C$, then the bifunction $f: C \times C \rightarrow \mathbb{R}$, defined by

$$
f(x, y)=\langle A(x), y-x\rangle, \forall x, y \in C
$$

is also monotone on $C$.

Lemma 2.1. Let $\alpha(t):[0, \infty) \rightarrow(0, \infty)$, and let $u(t):[0, \infty) \rightarrow \mathbb{R}^{m}$ be continuous functions satisfying $\int_{0}^{\infty} \alpha(t) d t=\infty$ and $\lim _{t \rightarrow \infty} u(t)=a$. Then,

$$
\frac{\int_{0}^{\infty} \alpha(t) u(t) d t}{\int_{0}^{\infty} \alpha(t) d t}=a .
$$

Proof. Obviously, it is sufficient to consider the case $m=1$ and $a=0$. If $\int_{0}^{\infty} \alpha(t) u(t) \mathrm{d} t<\infty$, then we have

$$
\frac{\int_{0}^{\infty} \alpha(t) u(t) \mathrm{d} t}{\int_{0}^{\infty} \alpha(t) \mathrm{d} t}=0
$$


which is the desired result. In the opposite case, applying Lhospital's rule, we get

$$
\begin{aligned}
\lim _{A \rightarrow \infty} \frac{\int_{0}^{A} \alpha(t) u(t) \mathrm{d} t}{\int_{0}^{A} \alpha(t) \mathrm{d} t} & =\lim _{A \rightarrow \infty} \frac{\alpha(A) u(A)}{\alpha(A)} \\
& =\lim _{A \rightarrow \infty} u(A)=a
\end{aligned}
$$

Lemma 2.2. Let $\alpha(t):[0, \infty) \rightarrow(0, \infty)$, and let $u(t):[0, \infty) \rightarrow C$ be continuous functions. Define

$$
z(t)=\frac{\int_{0}^{t} \alpha(\tau) u(\tau) d \tau}{\int_{0}^{t} \alpha(\tau) d \tau}
$$

Then, $z(t) \in C$ for all $t \geq 0$.

Proof. We have

$$
\int_{0}^{t} \alpha(\tau) u(\tau) \mathrm{d} \tau=\lim _{n \rightarrow \infty} \frac{t}{n} \sum_{i=1}^{n}\left(\alpha\left(\frac{i t}{n}\right) u\left(\frac{i t}{n}\right)\right) \quad \text { and } \int_{0}^{t} \alpha(\tau) \mathrm{d} \tau=\lim _{n \rightarrow \infty} \frac{t}{n} \sum_{i=1}^{n} \alpha\left(\frac{i t}{n}\right) .
$$

Hence

$$
z(t)=\lim _{n \rightarrow \infty} \frac{\sum_{i=1}^{n}\left(\alpha\left(\frac{i t}{n}\right) u\left(\frac{i t}{n}\right)\right)}{\sum_{i=1}^{n} \alpha\left(\frac{i t}{n}\right)} .
$$

Since $C$ is convex, $u(t) \in C$ for all $t \geq 0$, we have

$$
\frac{\sum_{i=1}^{n}\left(\alpha\left(\frac{i t}{n}\right) u\left(\frac{i t}{n}\right)\right)}{\sum_{i=1}^{n} \alpha\left(\frac{i t}{n}\right)} \in C \quad \forall n .
$$

Note that $C$ is closed. Taking the limit as $n \rightarrow \infty$, we have $z(t) \in C$.

Assumption 2.1. In this paper, we consider problem $\operatorname{VI}(A, C)$ under the following assumptions:

(A1) The mapping $A$ is monotone on $C$;

(A2) The mapping $A$ is continuous on $C$;

(A3) The solution set $\operatorname{Sol}(A, C)$ of $\operatorname{VI}(A, C)$ is not empty.

Lemma 2.3. [6] Under Assumption 2.1, it holds that

(i) $\operatorname{Sol}(A, C)=\{x \in C:\langle A(y), y-x\rangle \geq 0 \forall y \in C\}$;

(ii) The solution set of $\operatorname{VI}(A, C)$ is closed and convex.

\section{MAin RESUlts}

For solving $\operatorname{VI}(A, C)$, we consider the following dynamical system:

$$
\left\{\begin{array}{l}
x(0) \in C \\
\dot{x}(t)=\delta\left\{P_{C}\left(x(t)-\frac{\alpha(t)}{\max \{1 ;\|A(x(t))\|\}} A(x(t))\right)-x(t)\right\} .
\end{array}\right.
$$

Theorem 3.1. Suppose that Assumption 2.1 holds and $\alpha(t):[0, \infty) \rightarrow(0, \infty)$ is a continuous function satisfying

$$
\int_{0}^{\infty} \alpha(t) \mathrm{d} t=\infty, \int_{0}^{\infty} \alpha(t)^{2} \mathrm{~d} t<\infty
$$


Let $x(t)$ be the solution of (3.1), and denote

$$
z(t)=\frac{\int_{t_{0}}^{t} \lambda(\tau) x(\tau) \mathrm{d} \tau}{\int_{t_{0}}^{t} \lambda(\tau) \mathrm{d} \tau}, \text { where } \lambda(t)=\frac{\alpha(t)}{\max \{1 ;\|A(x(t))\|\}}, t_{0} \in[0, \infty) .
$$

Then,

$$
\lim _{t \rightarrow \infty} z(t)=z^{*} \in \operatorname{Sol}(A, C) .
$$

Proof. Without loss of generality, we may assume that $t_{0}=0$. We divide the proofs into several steps.

Step 1: We prove that $x(t) \in C$ for all $t \geq 0$. Denote

$$
y(t):=P_{C}\left(x(t)-\frac{\alpha(t)}{\max \{1 ;\|A(x(t))\|\}} A(x(t))\right) .
$$

Obviously, $y(t)$ is a continuous function and $y(t) \in C$ for all $t \geq 0$. It holds that

$$
\dot{x}(t)+\delta x(t)=\delta y(t) .
$$

Thus,

$$
\begin{aligned}
x(t) & =e^{-\delta t}\left(\int_{0}^{t} \delta y(s) e^{\delta s} \mathrm{~d} s+x(0)\right) \\
& =\left(1-e^{-\delta t}\right) \frac{1}{e^{\delta t}-1} \int_{0}^{t} \delta y(s) e^{\delta s} \mathrm{~d} s+e^{-\delta t} x(0) .
\end{aligned}
$$

Since $C$ is convex and $x(0) \in C$, it is sufficient to show that

$$
\frac{1}{e^{\delta t}-1} \int_{0}^{t} \delta y(s) e^{\delta s} \mathrm{~d} s \in C, \quad \forall t>0
$$

We have

$$
\frac{1}{e^{\delta t}-1} \int_{0}^{t} \delta y(s) e^{\delta s} \mathrm{~d} s=\lim _{n \rightarrow \infty} \sum_{i=0}^{n-1} \frac{\delta t e^{\delta \frac{i t}{n}}}{n\left(e^{\delta t}-1\right)} y\left(\frac{i t}{n}\right)
$$

Note that

$$
\sum_{i=0}^{n-1} \frac{\delta t e^{\delta \frac{i t}{n}}}{n\left(e^{\delta t}-1\right)}=\frac{\delta t}{n\left(e^{\frac{\delta t}{n}}-1\right)}<1
$$

Hence $\frac{\delta t e^{\delta \frac{i t}{n}}}{n\left(e^{\delta t}-1\right)} \in(0,1)$ for all $i=0, \ldots, n-1$. Moreover,

$$
\lim _{n \rightarrow \infty} \frac{\delta t}{n\left(e^{\frac{\delta t}{n}}-1\right)}=1
$$

and $y\left(\frac{i t}{n}\right) \in C$, which is closed and convex. Hence,

$$
\frac{1}{e^{\delta t}-1} \int_{0}^{t} \delta y(s) e^{\delta s} \mathrm{~d} s \in C
$$

Step 2: We prove that, for any solution $x^{*} \in \operatorname{Sol}(A, C)$, there exists the limit

$$
\lim _{t \rightarrow \infty}\left\|x(t)-x^{*}\right\| \text {. }
$$

Let

$$
V(t)=\frac{1}{2}\left\|x(t)-x^{*}\right\|^{2}
$$


We have

$$
\begin{aligned}
\dot{V}(t) & =\left\langle x(t)-x^{*}, \dot{x}(t)\right\rangle \\
& =\delta\left\langle x(t)-x^{*}, P_{C}\left(x(t)-\frac{\alpha(t)}{\max \{1 ;\|A(x(t))\|\}} A(x(t))\right)-x(t)\right\rangle \\
& =\delta\left\{\left\langle x(t)-x^{*}, P_{C}\left(x(t)-\frac{\alpha(t)}{\max \{1 ;\|A(x(t))\|\}} A(x(t))\right)-x^{*}\right\rangle-\left\|x(t)-x^{*}\right\|^{2}\right\} \\
& \leq \frac{\delta}{2}\left\{\left\|P_{C}\left(x(t)-\frac{\alpha(t)}{\max \{1 ;\|A(x(t))\|\}} A(x(t))\right)-x^{*}\right\|^{2}-\left\|x(t)-x^{*}\right\|^{2}\right\} .
\end{aligned}
$$

On the other hand, it holds that

$$
\begin{aligned}
& \left\|P_{C}\left(x(t)-\frac{\alpha(t)}{\max \{1 ;\|A(x(t))\|\}} A(x(t))\right)-x^{*}\right\|^{2} \\
= & \left\|P_{C}\left(x(t)-\frac{\alpha(t)}{\max \{1 ;\|A(x(t))\|\}} A(x(t))\right)-P_{C}\left(x^{*}\right)\right\|^{2} \\
\leq & \left\|\left(x(t)-x^{*}\right)-\frac{\alpha(t)}{\max \{1 ;\|A(x(t))\|\}} A(x(t))\right\|^{2} \\
\leq & \left\|x(t)-x^{*}\right\|^{2}-\frac{2 \alpha(t)}{\max \{1 ;\|A(x(t))\|\}}\left\langle A(x(t)), x(t)-x^{*}\right\rangle+\alpha(t)^{2}
\end{aligned}
$$

Since $x^{*} \in \operatorname{Sol}(A, C), x(t) \in C$ and $A$ is monotone, we get from (3.3) that

$$
\left\|P_{C}\left(x(t)-\frac{\alpha(t)}{\max \{1 ;\|A(x(t))\|\}} A(x(t))\right)-x^{*}\right\|^{2} \leq\left\|x(t)-x^{*}\right\|^{2}+\alpha(t)^{2} .
$$

Combining (3.2) and (3.4), we obtain

$$
\dot{V}(t) \leq \frac{\delta}{2} \alpha(t)^{2}
$$

\section{Denote}

$$
w(t):=\max \{\dot{V}(t) ; 0\} ; \quad v(t):=\max \{-\dot{V}(t) ; 0\}
$$

We have

$$
\dot{V}(t)=w(t)-v(t)
$$

hence

$$
V(t)=\int_{0}^{t} w(\tau) \mathrm{d} \tau-\int_{0}^{t} v(\tau) \mathrm{d} \tau+V(0)
$$

On the other hand, since $v(t) \geq 0,0 \leq w(t) \leq \dot{V}(t) \leq \frac{\delta \alpha(t)^{2}}{2}$ and $\int_{t=0}^{\infty} \alpha(t)^{2} \mathrm{~d} t<\infty$, it holds that

$$
\lim _{t \rightarrow \infty} \int_{0}^{t} v(\tau) \mathrm{d} \tau=c \in \overline{\mathbb{R}}, \quad \lim _{t \rightarrow \infty} \int_{0}^{t} w(\tau) \mathrm{d} \tau=d \in \mathbb{R}
$$

Combining (3.5), (3.6) and the fact that $V(t) \geq 0$, we deduce that there exists the limit

$$
\lim _{t \rightarrow \infty} V(t) \in \mathbb{R} \text {. }
$$

Let $u(t)=P_{S o l(A, C)}(x(t))$. 
Step 3: We prove that

$$
\lim _{t \rightarrow \infty} u(t)=\hat{u} \in \operatorname{Sol}(A, C) .
$$

Let $u \in \operatorname{Sol}(A, C)$, and $W(t)=\frac{1}{2}\|x(t)-u\|^{2}$. Similarly to Step 2, we have

$$
\dot{W}(t) \leq \frac{\delta}{2} \alpha(t)^{2}
$$

For all $t, h \geq 0$, it holds that

$$
\begin{aligned}
W(t+h) & =W(t)+\int_{t}^{t+h} \dot{W}(\tau) \mathrm{d} \tau \\
& \leq W(t)+\int_{t}^{t+h} \frac{\delta}{2} \alpha(\tau)^{2} \mathrm{~d} \tau .
\end{aligned}
$$

Hence,

$$
\|x(t+h)-u\|^{2} \leq\|x(t)-u\|^{2}+\int_{t}^{t+h} \delta \alpha(\tau)^{2} \mathrm{~d} \tau, \quad \forall u \in \operatorname{Sol}(A, C), \forall t, h \in[0, \infty) .
$$

Thus,

$$
\|x(t+h)-u(t)\|^{2} \leq\|x(t)-u(t)\|^{2}+\int_{t}^{t+h} \delta \alpha(\tau)^{2} \mathrm{~d} \tau \quad \forall t, h \in[0, \infty) .
$$

On the other hand, since $u(t+h)=P_{\text {Sol }(A, C)}(x(t+h))$ and $\frac{1}{2}(u(t+h)+u(t)) \in C$, we get

$$
\|x(t+h)-u(t+h)\|^{2} \leq\left\|x(t+h)-\frac{1}{2}(u(t+h)+u(t))\right\|^{2} .
$$

Hence

$$
\begin{aligned}
\|u(t+h)-u(t)\|^{2}= & \|u(t+h)-x(t+h)+x(t+h)-u(t)\|^{2} \\
= & 2\|x(t+h)-u(t+h)\|^{2}-4\left\|x(t+h)-\frac{1}{2}(u(t+h)+u(t))\right\|^{2} \\
& +2\|x(t+h)-u(t)\|^{2} \\
\leq & 2\|x(t+h)-u(t)\|^{2}-2\|x(t+h)-u(t+h)\|^{2} \\
\leq & 2\|x(t)-u(t)\|^{2}-2\|x(t+h)-u(t+h)\|^{2}+2 \int_{t}^{t+h} \delta \alpha(\tau)^{2} \mathrm{~d} \tau .
\end{aligned}
$$

We have

$$
\|x(t+h)-u(t+h)\|^{2} \leq\|x(t)-u(t)\|^{2}+\int_{t}^{t+h} \delta \alpha(\tau)^{2} \mathrm{~d} \tau \forall t, h \geq 0 .
$$

It implies that

$$
\limsup _{\tau \rightarrow \infty}\|x(\tau)-u(\tau)\|^{2} \leq\|x(t)-u(t)\|^{2}+\int_{t}^{\infty} \delta \alpha(\tau)^{2} \mathrm{~d} \tau \forall t \geq 0
$$

or

$$
\limsup _{\tau \rightarrow \infty}\|x(\tau)-u(\tau)\|^{2} \leq \liminf _{t \rightarrow \infty}\|x(t)-u(t)\|^{2}+\liminf _{t \rightarrow \infty} \int_{t}^{\infty} \delta \alpha(\tau)^{2} \mathrm{~d} \tau .
$$

Since $\int_{t}^{\infty} \delta \alpha(\tau)^{2} \mathrm{~d} \tau<\infty$, we have

$$
\liminf _{t \rightarrow \infty} \int_{t}^{\infty} \delta \alpha(\tau)^{2} \mathrm{~d} \tau=0
$$


Thus, there exists the $\operatorname{limit}_{t \rightarrow \infty}\|x(t)-u(t)\|^{2}$. Combining this and (3.7), we deduce that there exists the $\operatorname{limit}_{\lim _{t \rightarrow \infty}} u(t)=\hat{u}$. But $u(t) \in \operatorname{Sol}(A, C)$ and $\operatorname{Sol}(A, C)$ is closed, it implies that $\hat{u} \in \operatorname{Sol}(A, C)$.

Step 4: We prove that $z(t) \rightarrow \hat{u} \in \operatorname{Sol}(A, C)$.

Let $x \in C$ be an arbitrary point. Denote $U(t):=\frac{1}{2}\|x(t)-x\|^{2}$. Applying arguments similar to (3.2) and (3.3), we have

$$
\begin{aligned}
\dot{U}(t) & \leq \delta \lambda(t)\langle A(x(t)), x-x(t)\rangle+\frac{\delta}{2} \alpha(t)^{2} \\
& \leq \delta \lambda(t)\langle A(x), x-x(t)\rangle+\frac{\delta}{2} \alpha(t)^{2}
\end{aligned}
$$

where

$$
\lambda(t)=\frac{\alpha(t)}{\max \{1 ;\|A(x(t))\|\}} .
$$

The last inequality comes from the monotonicity of $A$. From Step 2, it implies that the function $x(t)$ is bounded, so are the functions $\|A(x(t))\|$ and $z(t)$. There exists $M>0$ such that

$$
\|A(x(t))\| \leq M \quad \forall t \geq 0 .
$$

We have

$$
\int_{0}^{\infty} \lambda(t) \mathrm{d} t \geq \int_{0}^{\infty} \frac{\alpha(t)}{\max \{1 ; M\}} \mathrm{d} t=\infty .
$$

Integrating both sides of (3.8), we get

$$
\frac{\int_{0}^{t} \dot{U}(\tau) \mathrm{d} \tau}{\int_{0}^{t} \lambda(\tau) \mathrm{d} \tau} \leq \delta\left\langle A(x), x-\frac{\int_{0}^{t} \lambda(\tau) x(\tau) \mathrm{d} \tau}{\int_{0}^{t} \lambda(\tau) \mathrm{d} \tau}\right\rangle+\frac{\delta}{2} \frac{\int_{0}^{t} \alpha(\tau)^{2} \mathrm{~d} \tau}{\int_{0}^{t} \lambda(\tau) \mathrm{d} \tau},
$$

or

$$
\frac{U(t)-U(0)}{\int_{0}^{t} \lambda(\tau) \mathrm{d} \tau} \leq \delta\langle A(x), x-z(t)\rangle+\frac{\delta}{2} \frac{\int_{0}^{t} \alpha(\tau)^{2} \mathrm{~d} \tau}{\int_{0}^{t} \lambda(\tau) \mathrm{d} \tau}, \forall t \geq 0 .
$$

Suppose by contrary that $z(t) \nrightarrow \hat{u}$. Then, there exist $\varepsilon>0$ and a sequence $\left\{t_{n}\right\} \in[0, \infty)$ such that $t_{n} \rightarrow \infty$ and $\left\|z\left(t_{n}\right)-\hat{u}\right\| \geq \varepsilon$ for all $n \geq 0$. But we have that $\left\{z\left(t_{n}\right)\right\}$ is bounded. Hence there exists a subsequence $\left\{z\left(t_{n_{i}}\right)\right\} \subset\left\{z\left(t_{n}\right)\right\}$ such that $z\left(t_{n_{i}}\right) \rightarrow \hat{z}$. From Lemma 2.2, we have $\hat{z} \in C$. In (4.4), letting $t=t_{n_{i}} \rightarrow \infty$, and using

$$
\int_{0}^{\infty} \lambda(t) \mathrm{d} t=\infty, \int_{0}^{\infty} \alpha(t)^{2} \mathrm{~d} t=\infty
$$

and the function $U(t)$ is bounded, we get

$$
\langle A(x), x-\hat{z}\rangle \geq 0 \forall x \in C .
$$

Using Lemma 2.3, we get $\hat{z} \in \operatorname{Sol}(A, C)$. From $u(t)=P_{\operatorname{Sol}(A, C)}(x(t))$ and $\hat{z} \in \operatorname{Sol}(A, C)$, it implies that

$$
\langle\hat{z}-u(t), x(t)-u(t)\rangle \leq 0
$$

Thus,

$$
\begin{aligned}
\langle\hat{z}-\hat{u}, x(t)-u(t)\rangle & =\langle\hat{z}-u(t), x(t)-u(t)\rangle+\langle u(t)-\hat{u}, x(t)-u(t)\rangle \\
& \leq\langle u(t)-\hat{u}, x(t)-u(t)\rangle \\
& \leq L\|u(t)-\hat{u}\|,
\end{aligned}
$$


where $L:=\max \{\|x(t)-u(t)\|\}<\infty$. It implies that

$$
\left\langle\hat{z}-\hat{u}, z\left(t_{n_{i}}\right)-\frac{\int_{0}^{t_{n_{i}}} \lambda(\tau) u(\tau) \mathrm{d} \tau}{\int_{0}^{t_{n_{i}}} \lambda(\tau) \mathrm{d} \tau}\right\rangle \leq L \frac{\int_{0}^{t_{n_{i}}} \lambda(\tau)\|u(\tau)-\hat{u}\| \mathrm{d} \tau}{\int_{0}^{t_{n_{i}}} \lambda(\tau) \mathrm{d} \tau}
$$

In (3.10), letting $i \rightarrow \infty$, and applying Lemma 2.1, we get $\|\hat{z}-\hat{u}\|^{2} \leq 0$, which is a contradiction.

\section{EXTENSION TO EQUILIBRIUM PROBLEMS}

Let $f: C \times C \rightarrow \mathbb{R}$ be a bifunction satisfying $f(x, x)=0$ for all $x \in C$. The equilibrium problem (EP) of $f$ on $C$ is to

$$
\text { find } x^{*} \in C \text { such that } f\left(x^{*}, y\right) \geq 0, \forall y \in C \text {. }
$$

It is well known that the equilibrium problem is an extension of the variational inequality. Moreover, it includes many other problems as special cases, such as fixed point problems, Nash equilibrium problems, optimization problems. In this section, we will extend our algorithm to EPs. Denote by $\operatorname{Sol}(f, C)$ the solution set of $\operatorname{EP}(f, C)$.

Assumption 4.1. We consider $\operatorname{EP}(f, C)$ under the following assumptions:

(B1) the bifunction $f$ is monotone on $C$;

(B2) for all $x \in C$, the function $f(x,$.$) is convex, subdifferentiable and lower semicontinuous;$ the function $f(., x)$ is upper semicontinuous on $C$;

(B3) the mapping $x \mapsto \partial f(x,).(x)$ is bounded on bounded subsets of $C$;

(B4) the solution set $\operatorname{Sol}(f, C)$ is not empty.

Lemma 4.1. [6] Under Assumption 4.1, it holds that

(i) $\operatorname{Sol}(f, C)=\{y \in C: f(x, y) \leq 0 \forall x \in C\}$,

(ii) $\operatorname{Sol}(f, C)$ is closed and convex.

To solve $\operatorname{EP}(f, C)$, we propose the following dynamical system:

$$
\left\{\begin{array}{l}
x(0) \in C \\
\dot{x}(t)=\delta\left\{P_{C}\left(x(t)-\frac{\alpha(t)}{\max \{1 ; w(t)\}} w(t)\right)-x(t)\right\}, \\
\text { where } w(t) \in \partial f(x(t), .)(x(t)) .
\end{array}\right.
$$

Theorem 4.1. Suppose that Assumption 4.1 holds and $\alpha(t):[0, \infty) \rightarrow(0, \infty)$ is a continuous function satisfying

$$
\int_{0}^{\infty} \alpha(t) \mathrm{d} t=\infty, \int_{0}^{\infty} \alpha(t)^{2} \mathrm{~d} t<\infty .
$$

Let $x(t)$ be the solution of (4.1), and

$$
z(t)=\frac{\int_{t_{0}}^{t} \lambda(\tau) x(\tau) \mathrm{d} \tau}{\int_{t_{0}}^{t} \lambda(\tau) \mathrm{d} \tau}, \text { where } \lambda(t)=\frac{\alpha(t)}{\max \{1 ; w(t)\}}, t_{0} \geq 0
$$

Then,

$$
\lim _{t \rightarrow \infty} z(t)=z^{*} \in \operatorname{Sol}(f, C)
$$


Proof. Without loss of generality, we may assume that $t_{0}=0$. Let $x^{*} \in \operatorname{Sol}(f, C)$. Denote

$$
H(t):=\frac{1}{2}\left\|x(t)-x^{*}\right\|^{2} .
$$

Similarly to the proof of Theorem 3.1, we have $x(t) \in C$ for all $t \geq 0$ and

$$
\dot{H}(t) \leq \frac{\delta \alpha(t)}{\max \{1 ;\|w(t)\|\}}\left\langle w(t), x^{*}-x(t)\right\rangle+\frac{\delta}{2} \alpha(t)^{2} .
$$

From the definition of $w(t)$, it implies that

$$
\begin{aligned}
\left\langle w(t), x^{*}-x(t)\right\rangle & \leq f\left(x(t), x^{*}\right)-f(x(t), x(t)) \\
& =f\left(x(t), x^{*}\right) \\
& \leq 0 .
\end{aligned}
$$

The last inequality comes from the facts that $x(t) \in C$ and $f$ is monotone on $C$. Combining (4.2) and (4.3), we obtain

$$
\dot{H}(t) \leq \frac{\delta}{2} \alpha(t)^{2}
$$

which implies that there exists the limit $\lim _{t \rightarrow \infty} H(t)$. The functions $x(t)$ and $z(t)$ are bounded. From Assumption 4.1, it implies that the function $w(t)$ is bounded. Hence, we have

$$
\int_{0}^{\infty} \lambda(t) \mathrm{d} t=\infty
$$

Let $u(t)=P_{S o l(f, C)}(x(t))$. It is similar to Step 3 in the proof of Theorem 3.1 that

$$
\lim _{t \rightarrow \infty} u(t)=\hat{u} \in \operatorname{Sol}(f, C) .
$$

Now it remains to prove that

$$
\lim _{t \rightarrow \infty} z(t)=\hat{u} .
$$

Let $x \in C$ be an arbitrary point. Denote $K(t):=\frac{1}{2}\|x(t)-x\|^{2}$. Using the definition of $w(t)$ and the monotonicity of $f$, we have

$$
\begin{aligned}
\dot{K}(t) & \leq \delta \lambda(t)\langle w(t), x-x(t)\rangle+\frac{\delta}{2} \alpha(t)^{2} \\
& \leq \delta \lambda(t) f(x(t), x)+\frac{\delta}{2} \alpha(t)^{2} \\
& \leq-\delta \lambda(t) f(x, x(t))+\frac{\delta}{2} \alpha(t)^{2} .
\end{aligned}
$$

Integrating both sides of the last inequality, and taking into account the convexity of the function $f(x,$.$) , we get$

or

$$
\begin{aligned}
\frac{\int_{0}^{t} \dot{K}(\tau) \mathrm{d} \tau}{\int_{0}^{t} \lambda(\tau) \mathrm{d} \tau} & \leq-\delta \frac{\int_{0}^{t} \lambda(\tau) f(x, x(\tau)) \mathrm{d} \tau}{\int_{0}^{t} \lambda(\tau) \mathrm{d} \tau}+\frac{\delta}{2} \frac{\int_{0}^{t} \alpha(\tau)^{2} \mathrm{~d} \tau}{\int_{0}^{t} \lambda(\tau) \mathrm{d} \tau} \\
& \leq-\delta f\left(x, \frac{\int_{0}^{t} \lambda(\tau) x(\tau) \mathrm{d} \tau}{\int_{0}^{t} \lambda(\tau) \mathrm{d} \tau}\right)+\frac{\delta}{2} \frac{\int_{0}^{t} \alpha(\tau)^{2} \mathrm{~d} \tau}{\int_{0}^{t} \lambda(\tau) \mathrm{d} \tau}
\end{aligned}
$$

$$
\frac{K(t)-K(0)}{\int_{0}^{t} \lambda(\tau) \mathrm{d} \tau} \leq-\delta f(x, z(t))+\frac{\delta}{2} \frac{\int_{0}^{t} \alpha(\tau)^{2} \mathrm{~d} \tau}{\int_{0}^{t} \lambda(\tau) \mathrm{d} \tau} .
$$


Suppose by contrary that $z(t) \nrightarrow \hat{u}$. Then, there exist $\varepsilon>0$ and a sequence $\left\{t_{n}\right\} \in[0, \infty)$ such that $t_{n} \rightarrow \infty$ and $\left\|z\left(t_{n}\right)-\hat{u}\right\| \geq \varepsilon$ for all $n \geq 0$. But we have $\left\{z\left(t_{n}\right)\right\}$ is bounded. Hence there exists a subsequence $\left\{z\left(t_{n_{i}}\right)\right\} \subset\left\{z\left(t_{n}\right)\right\}$ such that $z\left(t_{n_{i}}\right) \rightarrow \hat{z}$. From Lemma 2.2, we have $\hat{z} \in C$. In (4.4), letting $t=t_{n_{i}} \rightarrow \infty$, and using the lower semicontinuous of $f(x,$.$) , we have$

$$
f(x, \hat{z}) \leq 0, \forall x \in C .
$$

From Lemma 4.1, it follows that $\hat{z} \in \operatorname{Sol}(f, C)$. Applying the arguments similar to the ones in Step 4 of the proof of Theorem 3.1, we have $\hat{z}=\hat{u}$. This is a contradiction. Hence, $z(t) \rightarrow \hat{u}$.

\section{CONCLUSION}

We introduced a new method for solving variational inequalities. First, we considered a projected-type dynamical system associated with the variational inequality. Under the assumptions that the involving mapping is monotone, we proved that the ergodic function of the solution of this dynamical system converges to a solution of the variational inequality. The new method can be considered as a continuous version of the discrete one, introduced by Iiduka [9]. The new approach not only provides a new perspective on the ergodic algorithm but also removes a convergence condition of the existing one.

\section{Acknowledgments}

This work was supported by Vietnam Ministry of Education and Training under grant number B2020-BKA- 21-CTTH.

\section{REFERENCES}

[1] P.K. Anh, T.N. Hai, Splitting extragradient-like algorithms for strongly pseudomonotone equilibrium problems, Numer. Algo. 76 (2017), 67-91.

[2] T.Q. Bao, P.Q. Khanh, A projection-type algorithm for pseudomonotone nonlipschitzian multi-valued variational inequalities, In: Eberhard A., Hadjisavvas N., Luc D.T. (eds) Generalized Convexity, Generalized Monotonicity and Applications, Nonconvex Optimization and Its Applications, vol 77, Springer, Boston, 2005.

[3] J.Y. Bello Cruz, A.N. Iusem, An explicit algorithm for monotone variational inequalities, Optimization 61 (2012), 855-871.

[4] J.Y. Bello Cruz, A.N. Iusem, Convergence of direct methods for paramonotone variational inequalities, Comput. Optim. Appl. 46 (2010), 247-263.

[5] M. Tian, G. Xu, Inertial modified Tseng's extragradient algorithms for solving monotone variational inequalities and fixed point problems, J. Nonlinear Funct. Anal. 2020 (2020), Article ID 35.

[6] T.N. Hai, N.T. Vinh, Two new splitting algorithms for equilibrium problems, Rev. R. Acad. Cienc. Exactas Fís. Nat. Ser. A Math. RACSAM 111 (2017), 1051-1069.

[7] H. Iiduka, A new iterative algorithm for the variational inequality problem over the fixed point set of a firmly nonexpansive mapping, Optimization 59 (2010), 873-885.

[8] H. Iiduka, Fixed point optimization algorithm and its application to power control in CDMA data networks, Math. Program. 133(2012), 227-242.

[9] H. Iiduka, I. Yamada, An ergodic algorithm for the power-control games for CDMA data networks, J. Math. Model. Algorithms 8 (2009), 1-18.

[10] P.D. Khanh, P.T. Vuong, Modified projection method for strongly pseudomonotone variational inequalities, J. Glob. Optim. 58 (2014), 341-350.

[11] D. Kinderlehrer, G. Stampacchia, An Introduction to Variational Inequalities and Their Applications, Academic Press, New York, 1980.

[12] I.V. Konnov, Combined Relaxation Methods for Variational Inequalities, Springer, Berlin, 2000. 
[13] G.M. Korpelevich, The extragradient method for finding saddle points and other problems, Ekon. Mat. Metody. 12 (1976), 747-756.

[14] Y. Malitsky, Projected reflected gradient methods for monotone variational inequalities, SIAM J. Optim. 25 (2015), 502-520.

[15] S.Y. Cho, Strong convergence analysis of a hybrid algorithm for nonlinear operators in a Banach space, J. Appl. Anal. Comput. 8 (2018), 19-31.

[16] M.V. Solodov, B.F. Svaiter, A new projection method for monotone variational inequality problems, SIAM J. Control Optim. 37 (1999), 765-776.

[17] Y.J. Wang, N.H. Xiu , J.Z. Zhang, Modified extragradient method for variational inequalities and verification of solution existence, J. Optim. Theory Appl. 119 (2003), 167-183.

[18] J. Yang, H. Liu, Strong convergence result for solving monotone variational inequalities in Hilbert space, Numer. Algor. 80 (2019), 741-752.

[19] P.N. Anh, T.N. Hai, P.M. Tuan, On ergodic algorithms for equilibrium problems, J. Global Optim. 64 (2016), 179-195.

[20] J.K. Kim, P.N. Anh, T.N. Hai, The Bruck's ergodic iteration method for the Ky Fan inequality over the fixed point set, Int. J. Comput. Math. 94 (2017), 2466-2480.

[21] P.T. Vuong, The global exponential stability of a dynamical system for solving variational inequalities, Netw. Spatial Econ. (2019). https://doi.org/10.1007/s11067-019-09457-6.

[22] P.T. Vuong, J.J. Strodiot, A dynamical system for strongly pseudo-monotone equilibrium problems, J. Optim. Theory Appl. 185 (2020), 767-784.

[23] M. Pappalardo, M. Passacantando, Stability for equilibrium problems: from variational inequalities to dynamical systems, J. Optim. Theory Appl. 113 (2002), 567-582.

[24] A. Nagurney, D. Zhang, Projected dynamical systems and variational inequalities with applications, Kluwer Academic, Dordrecht, 1996.

[25] N.T.T. Ha, J.J. Strodiot, P.T. Vuong, On the global exponential stability of a projected dynamical system for strongly pseudomonotone variational inequalities, Opt. Lett. 12 (2018), 1625-1638.

[26] H.H. Bauschke, P.L. Combettes, Convex analysis and monotone operator theory in hilbert spaces, Springer, New York, 2011.

[27] F. Facchinei, J.-S. Pang, Finite-dimensional variational inequalities and complementarity problems, Springer, New York, 2003.

[28] R.T. Rockafellar, Convex Analysis, Princeton University Press, Princeton, New Jersey, 1970. 